\title{
Measuring constructs from the Consolidated Framework for Implementation Research in the context of increasing colorectal cancer screening at community health centers
}

\author{
Shuting Liang ${ }^{1 *}$, Michelle Kegler ${ }^{1}$, Michelle Carvalho ${ }^{1}$, Maria Fernandez ${ }^{2}$, Bryan Weiner ${ }^{3}$, Sara Jacobs ${ }^{4}$, \\ Rebecca Williams ${ }^{5}$, Betsy Risendal ${ }^{6,7}$, Letoynia Coombs ${ }^{6}$, Daniela Friedman ${ }^{8,9}$, Beth Glenn ${ }^{10,11,12}$, Shin-Ping Tu ${ }^{13}$ \\ From 7th Annual Conference on the Science of Dissemination and Implementation in Health \\ North Bethesda, MD, USA. 8-9 December 2014
}

\section{Background}

The Consolidated Framework for Implementation Research (CFIR) is a comprehensive meta-framework widely applied to implementation related studies. Yet, few have used validated measures to operationalize constructs in CFIR in real-life settings. In this study, we operationalized selected CFIR constructs in an assessment to identify factors influencing implementation of evidence-based practices for increasing colorectal cancer screening in Community Health Centers ( $\mathrm{CHC}$ ).

\section{Methods}

We selected 16 constructs from all five domains of CFIR. Measures were developed and tested in a cross-sectional survey with $\mathrm{CHCs}$ ' clinical staff and leaders respectively. We performed a separate confirmatory factor analysis (CFA) for measures with three or more items, computed inter-item consistency (Cronbach's alpha), inter-rater reliability (ICC) and agreement (rWG(J)) statistics, and assessed construct validity via inter-correlations among constructs at individual and organizational levels.

\section{Results}

A total of 277 individuals and $59 \mathrm{CHC}$ clinics were included in the analysis. CFA showed satisfactory structural validity $(\mathrm{CFI}>0.90, \quad \mathrm{TLI}>0.90, \quad \mathrm{SRMR}<0.08$, RMSEA $<0.08)$; all measures showed reasonable reliability (alpha $>0.70)$. The ICCs $(>0.1)$ and $\mathrm{rWG}(\mathrm{J})(>0.75)$ suggest

\footnotetext{
${ }^{1}$ Emory Prevention Research Center, Department of Behavioral Sciences and Health Education, Rollins School of Public Health, Emory University, Atlanta, GA 30322, USA

Full list of author information is available at the end of the article
}

it appropriate to aggregate individual responses by computing clinic means. Results also suggest good construct validity at both individual and clinic levels. Inner setting and process-related constructs are correlated with most variables across domains; correlations between outer setting and intervention characteristics and other domains vary more noticeably by construct.

\section{Implications}

Our study is one of the first to quantitatively measure constructs from all five domains of CFIR and demonstrate their psychometric properties. We depicted their inter-correlations at multiple levels, which set the foundation for establishing predictive models, causal pathways and developing interventions that target these factors. These findings could contribute to further development of the CFIR.
Authors' details
'Emory Prevention Research Center, Department of Behavioral Sciences and Health Education, Rollins School of Public Health, Emory University, Atlanta, GA 30322, USA. ${ }^{2}$ School of Public Health, University of Texas Health Science Center at Houston, Houston, TX 77030, USA. ${ }^{3}$ Department of Health Policy and Management, UNC Gillings School of Global Public Health, University of North Carolina at Chapel Hill, Chapel Hill, NC 27599, USA. ${ }^{4}$ Public Health Research Division, RTI International, Research Triangle Park, NC 27709, USA. ${ }^{5}$ Lineberger Comprehensive Cancer Center, School of Medicine, University of North Carolina at Chapel Hill, Chapel Hill, NC 27599, USA. 'University of Colorado Comprehensive Cancer Center, Aurora, CO 80045, USA.
${ }^{7}$ Department of Community and Behavioral Health, Colorado School of Public Health, Aurora, CO 80045, USA. ${ }^{8}$ Department of Health Promotion, Education, and Behavior, Arnold School of Public Health, University of South Carolina, Columbia, SC 29208, USA. ${ }^{9}$ Statewide Cancer Prevention and Control Program, Arnold School of Public Health, University of South Carolina, Columbia, SC 29208, USA. ${ }^{10}$ UCLA Kaiser Permanente Center for 
Health Equity, University of California Los Angeles, Los Angeles, CA 900956900, USA. ${ }^{11}$ Fielding School of Public Health, University of California Los Angeles, Los Angeles, CA 90095, USA. ${ }^{12}$ Jonsson Comprehensive Cancer Center, University of California Los Angeles, Los Angeles, CA 90095, USA.

${ }^{13}$ Department of Medicine, Virginia Commonwealth University, Richmond VA 23298, USA.

Published: 20 August 2015

doi:10.1186/1748-5908-10-S1-A10

Cite this article as: Liang et al:: Measuring constructs from the

Consolidated Framework for Implementation Research in the context of increasing colorectal cancer screening at community health centers.

Implementation Science 2015 10(Suppl 1):A10.

Submit your next manuscript to BioMed Central and take full advantage of:

- Convenient online submission

- Thorough peer review

- No space constraints or color figure charges

- Immediate publication on acceptance

- Inclusion in PubMed, CAS, Scopus and Google Scholar

- Research which is freely available for redistribution

Submit your manuscript at www.biomedcentral.com/submit 